Original Contribution

\title{
A CASE OF HYPERTRIGLYCERIDEMIA-INDUCEDSEVERE ACUTE PANCREATITIS - WHAT ELSE CAN BE DONE?
}

\author{
G. Minkov ${ }^{1 *}$, E. Dimitrov ${ }^{1}$, E. Enchev ${ }^{2}$, Kr. Halacheva $^{3}$, F. Hadjipetkov ${ }^{1}$,Iv. Dimitrov ${ }^{1}$, \\ St. Nikolov ${ }^{1}$, A. Petrov ${ }^{4}$, Y.Yovtchev ${ }^{1}$ \\ ${ }^{1}$ Department of Surgical Diseases and Anaesthesiology, Faculty of Medicine, Trakia University, \\ Stara Zagora, Bulgaria \\ ${ }^{2}$ Department of Clinical Pathology, Faculty of Medicine, Trakia University, Stara Zagora, Bulgaria \\ ${ }^{3}$ Department of Immunology and Biology, Faculty of Medicine, Trakia University, Stara Zagora, \\ Bulgaria \\ ${ }^{4}$ Department of General Surgery, Neurosurgery and Urology, Faculty of Medicine, Trakia University \\ Stara Zagora, Bulgaria
}

\begin{abstract}
Purpose: Acute pancreatitis (AP) often presents as complex diagnostic and management challenge to physicians, surgeons and radiologists caring for patients with the disease.

Methods: In this article we present a case of a young woman with AP induced by hypertriglyceridemia (HTGP) that runs on the background of diabetic ketoacidosis with unusual clinical picture and normal amylase.

Results and Conclusions: HTGP involves young people. Most of the cases can be presented with normal values of amylase and lipase, but the possibility of severe complications and the lack of specific therapies determine the need of early recognition. HTGP may need specific behaviour additional of generally accepted guidelines for the treatment of AP.
\end{abstract}

Key words: metabolic syndrome, diabetic ketoacidosis, pancreatitis

\section{INTRODUCTION}

The acute inflammation of the pancreas, which can possibly lead to a lethal outcome, is called acute pancreatitis (AP). It has a worldwide rising incidence over the past several decades [1]. Most cases are mild but there is a subdivision of patients with severe AP, where mortality may reach up to $30 \%$ [2]. Primary etiological factors are biliary stones and alcohol consumption. In up to $10 \%$ of the cases of acute pancreatitis the etiology is hypertriglyceridemia (HTG) [3]. It is considered that the levels of the triglycerides in the serum must be above $11 \mathrm{mmol} / 1$ to cause AP [4]. Increased levels of the triglycerides in the serum could have various causes like primary and secondary alterations of the lipids

Correspondence to: GeorgiMinkov, Department of Surgical Diseases and Anaesthesiology, Trakia University, 11 Armeiska Str., Stara Zagora, 6000, Bulgaria,e-mail:jogi85@abv.bg,phone: $+359882821208$ metabolism, alcoholism, diabetes mellitus and hypothyroidism [5]. The elevated levels of free fatty acids (FFA) and the formation of lysolecithin from lipoprotein substances in the pancreatic bed cause damage to acinar cells and microvascular membranes of the pancreas which is believed to be the mechanism behind this type of AP[6]. The clinical presentation of hypertriglyceridemia - associated pancreatitis (HTGP) can be atypical that's why the risk of complications could be higher [7]. We report a case of a female with severe AP induce by HTG.

\section{CASE PRESENTATION}

A 34-year-old obese female presented to the emergency department with a $2-3$ day history of abdominal pain, nausea, vomiting, progressive faintness and drowsiness. She had a medical history of diabetes mellitus type II for 6 years, hypothyroidism on $\mathrm{L}$ - Thyroxin therapy, and her gallbladder has been surgically removed. The patient denied any 
alcohol consumption. Physical examination revealed an alert patient (GCS - 15) with Kussmal acidotic breathing, subfebrile up to $37.7^{*} \mathrm{C}$, blood pressure of $90 / 60 \mathrm{mmHg}$, a pulse rate of $108 / \mathrm{min}$. Further examination revealed a soft, mildly tender abdomen with a dull pain particularly over the epigastric region on palpation. Relevant laboratory findings were: $\mathrm{pO} 2$ - $42.2 \mathrm{mmHg}$, WBC - $15.3 \times 10^{\wedge} 9 / 1$, blood glucose - $17.81 \mathrm{mmol} / \mathrm{l}$ and she was positive for ketones $(+)$, blood $(+)$, protein $(+)$ and glucose $(+)$ in urine. The patient was admitted to the Endocrinology department with diabetic ketoacidosis (DKA), she had normal amylase levels and normal ultrasound and $\mathrm{X}$ ray.

On the next day symptoms persisted and further tests were made. Relevant laboratory results were: CRP $315.8 \mathrm{mg} / \mathrm{l}$, triglycerides $12.06 \mathrm{mmol} / \mathrm{l}$, amylase and lipase were also normal.

On the third day a consultation with a surgeon was made which revealed a soft, mildly tender abdomen with a violent pain in the left half and the epigastric region on palpation and succutio renalis strong positive (++) in the left lumbar region. The tachydyspnea persisted. Relevant laboratory findings were: $\mathrm{K}+3.2 \mathrm{mmol} / 1, \mathrm{Na} 2+$ $128 \mathrm{mmol} / \mathrm{l}$, iCa++ $0.84 \mathrm{mmol} / 1$, CRP over
MINKOV G., et al.

$300 \mathrm{~g} / \mathrm{l}$ and normal amylase (Table 1). Ultrasound of the abdomen found free fluid in the abdomen between the intestinal loops and around the spleen, moderate hepatomegaly, diabetic nephropathy, surgically removed gallbladder without signs of dilated bill ducts. CT of the abdomen was made which showed signs of acute pancreatitis (Figure 1). The patient was transferred to the Department of Surgical Disease with signs of acute pancreatitis with normal amylase, respiratory and cardiovascular failure. SOFA and Modified Marshal Score were calculated and the disease was evaluated as severe (Table 1). We started therapeutic behavior according IAP/APA evidence - based guidelines and American College of Gastroenterology. At admission, aggressive hydration was started with $5-10 \mathrm{ml} / \mathrm{kg} / \mathrm{h}$ of isotonic crystalloid solution for the first $12-24 \mathrm{~h}$, analgesics were given for pain relief and Insulin - based on blood glucose level, measured on $3 \mathrm{~h}$. She was put on $0_{2}$ mask with levels set on $8-10 \mathrm{l} / \mathrm{min}$. From the first day in surgical department we started permanent infusion of insulin according levels of blood sugar and high dose of low density heparin. After the nausea and vomiting were resolved enteral feeding was commenced in first 24 hours. On the $10^{\text {th }}$ day the OF was resolved without need of IPV.

Table 1. Laboratory test results and calculated MMS (Modified Marshal Score) and SOFA (Sequential Organ Failure Assessment Score).

\begin{tabular}{|l|l|l|l|l|l|l|}
\hline & Day 1 & Day 2 & Day 5 & Day13 & Day 28 & Day 46 \\
\hline WBC & 15.3 & 12.1 & 10.1 & 11.26 & 7.5 & 6.8 \\
\hline HGB & 123 & 115 & 90 & 81 & 102 & 108 \\
\hline HCT & 0.371 & 0.342 & 0.271 & 0.265 & 0.279 & 0.332 \\
\hline Creatinine & 170 & 99 & 75 & 80 & 90 & 84 \\
\hline Urea & & 7.9 & 2.7 & 2.2 & 4.1 & 5.1 \\
\hline ALAT & & 11.1 & 13.6 & 19.3 & 16.2 & 9.7 \\
\hline ASAT & & 25.2 & 25.5 & 22.5 & 20.3 & 16.4 \\
\hline bilirubin & & & $1.8 / 10.4$ & $0.9 / 5.1$ & $0.9 / 4.7$ & $1.1 / 5.9$ \\
\hline glucose & 17.81 & 12.1 & 14.1 & 14.85 & 8.1 & 16.49 \\
\hline triglycerides & & 12.06 & 7.76 & 3.07 & 4.46 & 5.44 \\
\hline iCa++ & & & 0.84 & 0.82 & 1.14 & 1.17 \\
\hline $\boldsymbol{a}$-Amylase & & 83 & 56 & 80 & 207 & 290 \\
\hline CRP & & 315.8 & 295.5 & 168.6 & 56.4 & 4.9 \\
\hline LDH & & & 1415 & 606 & 714 & 503 \\
\hline pO'/Fi $\boldsymbol{O}^{2}$ & 148 & 158 & 164 & 410 & & \\
\hline Blood pressure & $90 / 60$ & $110 / 60$ & $110 / 70$ & $130 / 80$ & & \\
\hline $\begin{array}{l}\text { Body } \\
\text { temperature }\end{array}$ & 37.7 & 37.9 & 37.4 & 36.8 & & \\
\hline MMS & 3 & 3 & 3 & & & \\
\hline SOFA & 5 & 3 & 3 & & & \\
\hline
\end{tabular}




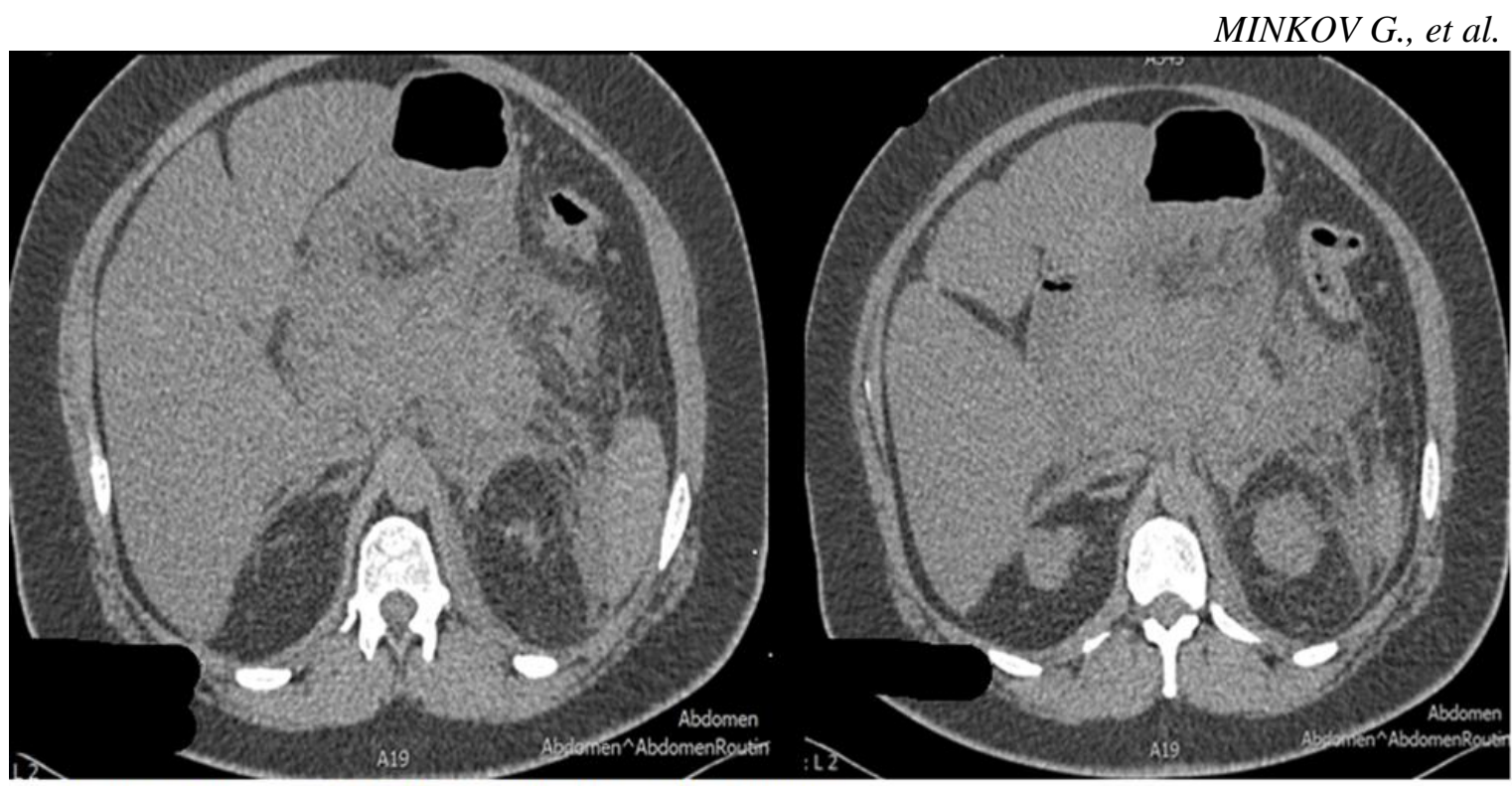

Figure 1. CT-scan on the 3th day of the disease development

On the $10^{\text {th }}$ day control contrast enhanced CTscan (CECT) was performed. We found acute necrotic collection (ANC) which spread over $50 \%$ of the parenchyma (Figure 2).
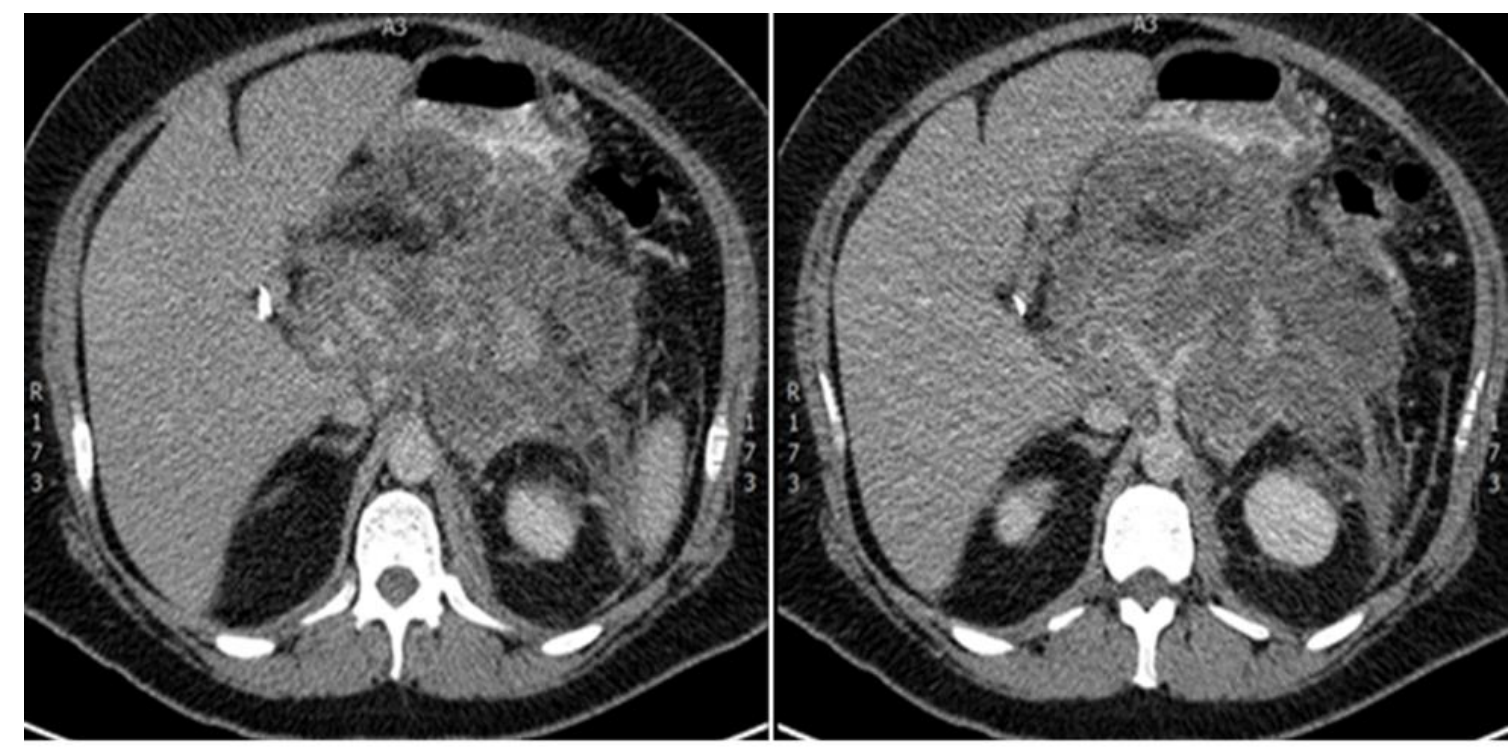

Figure 2. CECT on the 10th day of disease development

On the $19^{\text {th }}$ day the patient was discharged from the hospital without any complains and was asked to come back for control laboratory tests on $28^{\text {th }}$ day. The patient was discharged with good control of blood sugar, lower levels of serum triglycerides and significant excretory insufficiency.

On the $48^{\text {th }}$ day control CECT showed diffuse parenchymal enlargement, changed pancreatic density and sterile walled-off pancreatic necrosis WOPN (Figure 3).

\section{DISCUSSION}

Biliary stones and alcohol abuse are the major etiologic triggers of AP. HTG is responsible for AP in up to $10 \%$ of all cases [3]. The origins of HTG could be separated into two big groups: 1 . genetic factors: familial combined hyperlipidemia, familial HTG, familial dysbetalipoproteinemia, and familial chylomicronemia syndrome and 2.secondary factors: untreated/poorly controlled diabetes mellitus, pregnancy, and medications. HTGP involves young people, the normal values of amylase and lipase, the possibility of severe complications and the lack of specific therapies to determine the need for early recognition of this disease. 

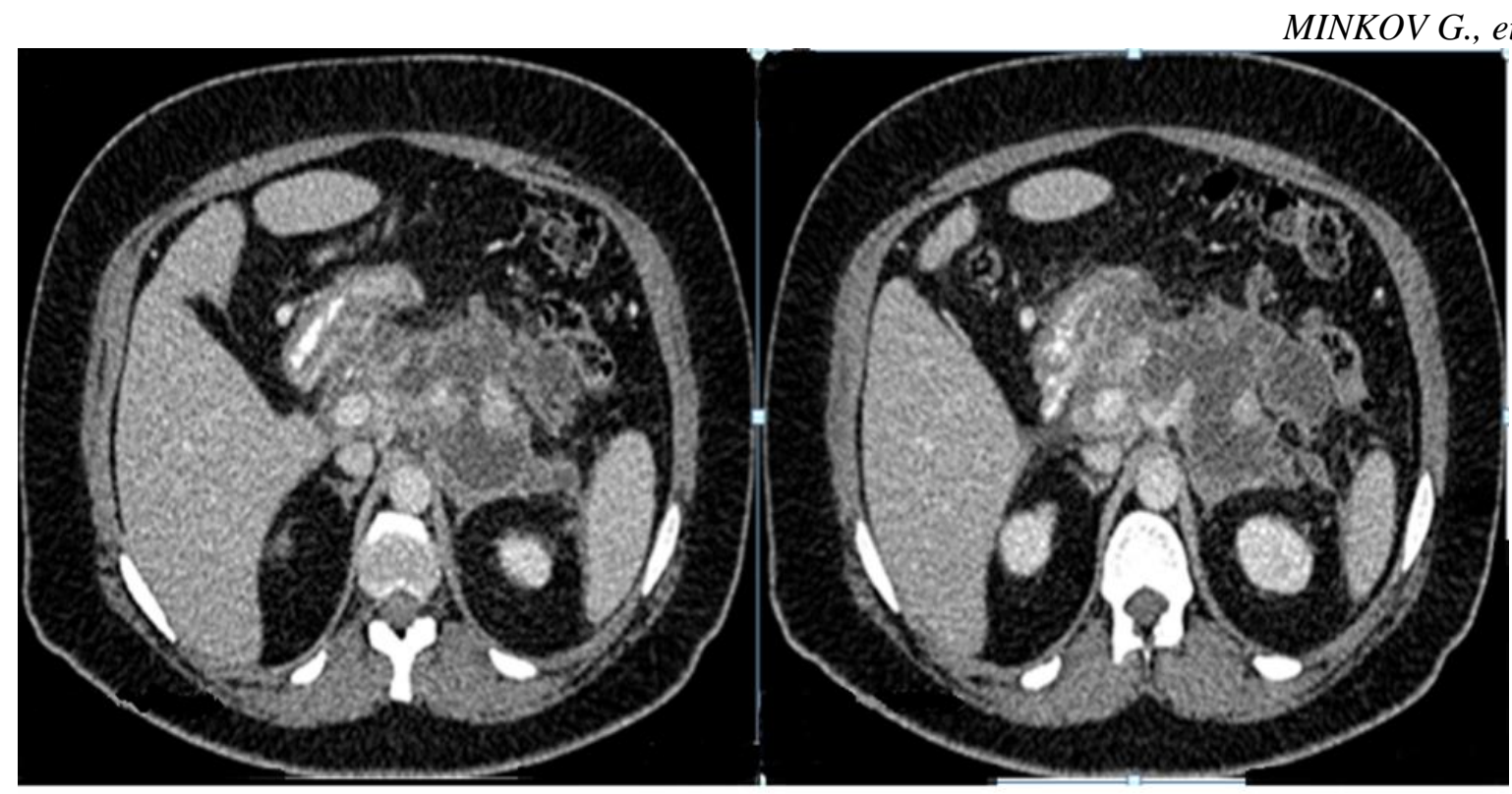

Figure 3. CECT on the 48th day of disease development

The exact pathogenic mechanism of how HTG can cause AP still remains unclear and is controversially discussed. The most popular theory is that the pancreatic lipase hydrolyzes the excess of triglycerides and this forms high concentrations of FFA which self-aggregate to micellar structures with detergent properties because they overwhelm the binding capacity of albumin. This promotes injury to the acinar cells and pancreatic capillaries. FFA toxicity is further enhanced by the acidic environment that is created by the resultant ischemia $[8,9]$.

The hyperchylomicronemia cause hyperviscosity in pancreatic capillaries that could possibly lead to ischemia and acidosis. Furthermore, there is proof that FFA can cause injury to the endothelium of the small vessels so AP could be linked with micro thrombosis and further ischemia [10], one possible reason for early pancreatic necrosis and complicated course.

The most common secondary factor for HTG, and eventually AP, is diabetes. Patients with uncontrolled/poorly controlled diabetes or in the setting of diabetic ketoacidosis (DKA) have higher serum triglycerides levels. Production of VLDL in the liver is accelerated because of the increased releasing of FFA in lipolysis in adipose tissue which is stimulated by the lack of insulin in patients in DKA.VLDL elimination form the plasma is reduced due to decreased activity of lipoprotein lipase in peripheral tissue which leads to HTG.

Crucial for diagnosing patients with HTGP are the high index of suspicion in appropriate clinical settings and the acquiring of serum triglycerides levels as close to the onset of pain during initial or recurrent attack(s) of AP as possible. Normoamylasemia is possible in about $60 \%$ of patients with HTGP. In the case that we present, amylase levels of the patient were within normal ranges too, which is considered to be due to disruption of the calorimetric method that cause interference with the in vitro measurement of the actual level of the amylase in the serum of the patient. Even though normoamylasemia may be present in patients with HTG, there should always be high index of suspicion for AP [11]. In $10-15 \%$ of the cases DKA may mask a coexisting AP because of ambiguous clinical presentation even though AP can trigger DKA itself. All the more, 24, 7 - 79, 0\% of the DKA cases are reported to have nonspecific elevations of serum amylase and/or lipase without clinical indications of AP. In patients with obscure unceasing abdominal pain is prudent to do further laboratory and imaging evaluations that's why CT scan of the abdomen is mandatory [11].

Despite the similar clinical course and the comparable risk of complications, patients with HTGP may need a specific approach. Timely infusion of insulin and heparin has been utilized to treat patients with HTGP [12-17]. For further treatment of HTGP, plasmapheresis, heparin infusion, and subcutaneous heparin were reported $[16,18$, 19]. Strict protocols for management of HTGP as none exist to date. 
We present a case of a young woman with HTGP that runs on the background of DKA with unusual clinical picture and normal enzymes in the serum. In the course of the disease, it is clear that imaging remains the "gold standard" for diagnosis. Respiratory and cardiac failures are associated with severe illness and high mortality in these patients with HTGP. In the presented case we definitely assume that the younger age of the patient with reserved compensatory possibilities has determined the favorable outcome of the disease. The unclear clinical picture and the lack of specific laboratory changes identified a delay therapeutic window. In this case, due to the elimination of the two most common causes of AP (biliary stones and alcohol), normal amylase, clinical and laboratory signs of DKA with poorly controlled diabetes; the patient was not considered having AP at first. In the next two days with no resolving abdominal pain, elevated inflammatory markers and serum triglycerides level CT was made which revealed the diagnosis of AP. The patient was discharged with improvement in clinical condition, lack of abdominal pain, decline in triglycerides levels in the serum, good response to treatment, and diet.

\section{CONCLUSION}

HTGP could be difficult for diagnose and may present with not so typical symptoms for the disease. The presentation of the disease may be with one of its complications. That is why it can be often mistaken for other disease or patients may end up in different departments depending on their clinical signs. It is important for those patients to be admitted to hospital and treatment to be started immediately. HTGP may need specific behavior additional of generally accepted guidelines for the treatment of AP. The pathophysiology remains poorly understood and seems to be very complex. However, this requires a thorough knowledge of underlying mechanisms and the patient's immunological state. It is most important to accurately identify patients with AP who are at risk of organ failure.

\section{REFERENCES}

1. Singla A, Csikesz NG, Simons JP et al. National hospital volume in acute pancreatitis: analysis of the Nationwide Inpatient Sample 1998-2006.HPB.2009. 11:391-397.
MINKOV G., et al.

2. Whitcomb DC. Clinical practice. Acute pancreatitis. N. Engl. J. Med. 2006. 354:2142-2150

3. Valdivielso P., Ramirez-Bueno A., Ewald N. Current knowledge of hypertriglyceridemic pancreatitis European Journal of Internal Medicine.2014. 25: 689-694.

4. Toskes PP. Hyperlipidemic pancreatitis. Gastroenterol.Clin.North Am.1990. 19:783791.

5. George Y, Khalid Z., Robert A. et al. Hypertriglyceridemia:its etiology, effects and treatment Canadian Medical Association Journal. 2007. 176:1113

6. Kimura W, Mossner J. Role of hypertriglyceridemia in the pathogenesis of experimental acute pancreatitis in rats. Int $J$ Pancreatol. 1996. 20:177-184.

7. Tsuang W, Navaneethan U, Ruiz L et al. Hypertriglyceridemic pancreatitis: presentation and management. AmJGastroenterol. 2009. 104:984-91.

8. Saharia P, Margolis S, Zuidema MD et al. Acute pancreatitis with hyperlipidemia: studies with an isolated perfused canine pancreas. Surgery. 1977. 82:60-67.

9. Kimura W, Mossner J. Role of hypertriglyceridemia in the pathogenesis of experimental acute pancreatitis in rats. Int. J. Pancreatol. 1996. 20:177-184.

10.Cuthbertson CM, Christophi C. Disturbances of the microcirculation in acute pancreatitis. Br. J. Surg. 2006. 93:518-530.

11.O'Keefe SJ. A guide to enteral access procedures and enteral nutrition. Nat Rev Gastroenterol Hepatol. 2009. 6:207-15.

12.Cole RP. Heparin treatment for severe hypertriglyceridemia in diabetic ketoacidosis. Arch Intern Med. 2009.169:1439-41.

13.Henderson SR, Maitland R, Mustafa OG, et al. Severe hypertriglyceridemia in Type 2 diabetes mellitus: beneficial effect of continuous insulin infusion. QJM. 2013. 106:355-9.

14. Alagozlu H, Cindoruk M, Karakan T et al. Heparin and insulin in the treatment of hypertriglyceridemia-induced severe acute pancreatitis. Dig Dis Sci. 2006.51:931-3.

15.Monga A, Arora A, Makkar RP et al. Hypertriglyceridemia-induced acute pancreatitis - treatment with heparin and insulin. Indian J Gastroenterol. 2003. 22:102-3. 
JD,

Mancell

Hypertriglyceridemia-induced

J. heparin. Am J Health Syst Pharm. 2012. 69:213-6.

17.Click B, Ketchum AM, Turner R et al. The role of apheresis in hypertriglyceridemiainduced acute pancreatitis: a systematic review. Pancreatology. 2015. 15:313-20
MINKOV G., et al. 18.Henzen C, Rock M, Schnieper C et al. Heparin and insulin in the treatment of acute hypertriglyceridemia-induced pancreatitis. Schweiz Med Wochenschr. 1999. 129:1242-8.

19.Berger Z, Quera R, Poniachik et al. Heparin and insulin treatment of acute pancreatitis caused by hypertriglyceridemia. Experience of 5 cases. Rev Med Chil. 2001. 129:13738. 